\title{
THE DETECTION OF CONCEALED TARGETS IN WOODLAND AREAS USING HYPERSPECTRAL IMAGERY
}

\author{
Santiago A. Rodriguez Gonzalez ${ }^{1, *}$, Michal Shimoni ${ }^{2}$, Javier Plaza $^{3}$, Antonio Plaza ${ }^{3}$, Ingmar Renhorn ${ }^{4}$, Jörgen Ahlberg $^{4,5}$ \\ ${ }^{1}$ National University of Cordoba (UNC), Cordoba, ARGENTINA - srodriguez@unc.edu.ar \\ ${ }^{2}$ Signal and Image Centre SIC-RMA, Brussels, Belgium \\ ${ }^{3}$ Hyperspectral Computing Laboratory, University of Extremadura, Cáceres, SPAIN \\ ${ }^{4}$ Glana Sensors AB, Linköping, SWEDEN \\ ${ }^{5}$ Computer Vision Laboratory, Linköping University, Linköping, SWEDEN
}

KEY WORDS: Hyperspectral Imaging, Target Detection, VIS-NIR, Woodland, Gradient Filter

\begin{abstract}
:
Recent innovations in microelectronic and semiconductor technology enable the creation of smaller and economical hyperspectral cameras. A filter combined camera with advanced scanning module is a game changer that extends the application of miniature hyperspectral imagers to many security domains. This work presents the assessment of the imager L4 from Glana Sensors to detect concealed targets in woodland areas. Several target detection methods were applied to a collection of scenes acquired under various illumination conditions and containing different materials. The potential and limitations of this new imaging device in the context of difficult target detection in forested area are evaluated and discussed.
\end{abstract}

\section{INTRODUCTION}

Detecting targets concealed in foliage or camouflage in large areas is a topic of interest in several security applications, such as search \& rescue, fighting cross-border crimes, or detecting enemies hidden in a forest. However, automatic detection and recognition of targets in forested areas is a very challenging research task, mainly due to the high intra-class variability of the background clutter including vegetation species, texture, and illumination.

With tens to hundreds contiguous spectral bands, hyperspectral imaging technology is now able to differentiate subtle spectral differences between land-cover objects, thus allowing for advanced material identification and separation techniques such as target detection (Q. Ling, 2019). Accordingly, hyperspectral technology has been successfully implemented for several applications areas, including, e.g., medicine, environment, public safety, and defense (Stefanou, Kerekes, 2010). Miniature sensors on-board unmanned aerial systems (UAS) are changing the operational capacity of security forces in forested landscapes, as they can acquire imagery of unreachable narrow and confined spaces (Petra et al., 2018). Traditionally, such systems are equipped with night-vision cameras and thermal sensors, but recent technological developments have enabled deployment of active sensing and multi/hyperspectral imagers (Waharte, Trigoni, 2018), (Jafari et al., 2014).

This paper presents the results of a study that evaluates the capability of advanced miniature hyperspectral imaging to detect concealed target in woodland areas. Specifically, it assesses the detection of concealed vehicle and small objects in forested zone using benchmarked detection algorithms, and discusses the limitation and the potential of the newly presented scanning technique.

\footnotetext{
${ }^{*}$ Corresponding author
}

\section{DATA COLLECTION}

\subsection{Hyperspectral Imager}

The miniature hyperspectral imager, L4 developed by Glana Sensors AB, was used in this study for spectral measurements. This advanced sensor is based on an exponentially variable filter (EVF) which is attached on, or in the direct proximity to the detector plane (Figure 1). The bandpass EVF filter allows the light to pass only in a narrow wavelength band, centered at a wavelength $\lambda_{c}$. This center wavelength varies over the filter as a function of the pixel position $(x, y)$. The center wavelength is a function of only one dimension, according to $\lambda_{c}=\lambda_{c}(x)$. As the EVF is mounted on, or very close to, the detector plane, the light registered by the sensor element at position $(x, y)$ will only contain wavelengths close to $\lambda_{c}(x, y)$. When the camera and the observed surface are static, each point on the object will thus be observed in a specific wavelength (Renhorn et al., 2016, Ahlberg et al., 2017). By rotating the camera, it can be used as an imaging spectrometer, as described in (Renhorn, Axelsson, 2019).

Exponentially Variable Filter Focal Plane Array

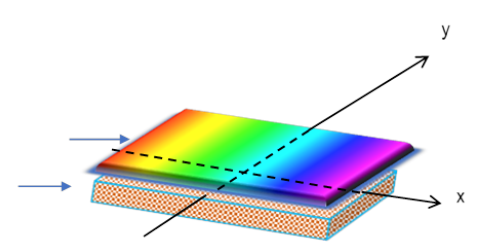

Figure 1. The EVF unit mounted on the detector plane of the miniature hyperspectral imager Glana-L4

\subsection{Spectral References}

The objects in the woodland scene were concealed with camouflage nets. For calibration and collection of reference spectra, the Glana-L4 was used to measure the camouflage nets at midsummer time, under clear sky conditions and known reflectance 
materials (panels). Specifically, a 5\% panel (Zenith SG3165) was placed behind the net and 20\% (Zenith SG3164) panel was positioned next to the net, as shown in Figure 2.

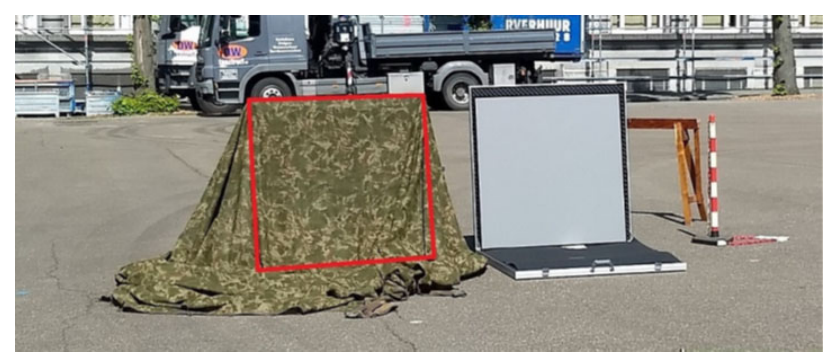

Figure 2. Collection of reference spectra.

\subsection{Image Acquisition}

Two sets of image data were collected in Vlaasmeer, a forested area at the North-East part of Belgium, using the Glana-L4. The camera was mounted on a tripod and scanned the field of view with horizontal motion and a frame rate (i.e. recording time) of 2 seconds for each independent band. In the first set of data, a concealed vehicle using two camouflage nets was placed in the shadow of the trees and scanned from near distance (Figure 3). This operational setting produced a common geometrical acquisition using UAS (Unmanned Aerial Systems), where the target covers most of the scene and only a limited amount of background pixels is available.

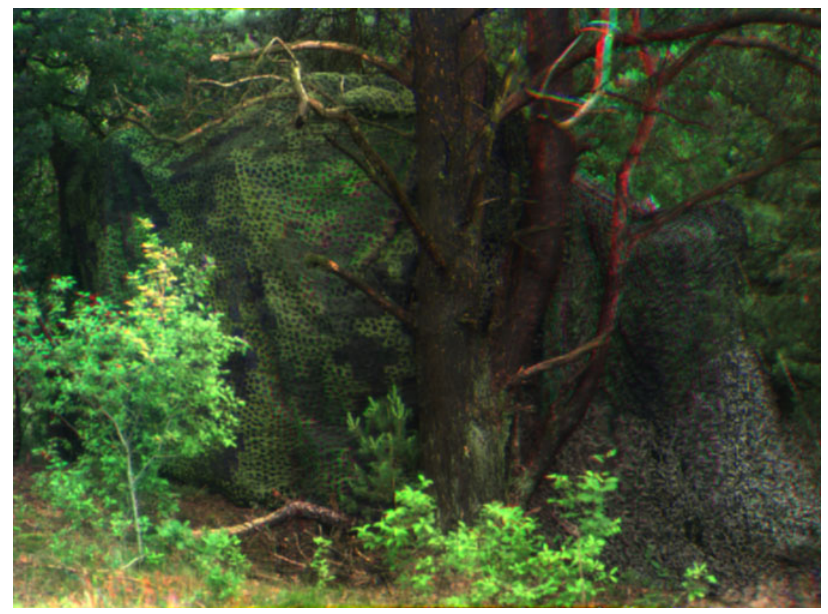

Figure 3. Concealed vehicle.

In the second set of data, two vertical metal objects were concealed using camouflage nets and positioned in front of a tree line and under direct illumination (Figure 4). Meteorological parameters such as ambient temperature, wind speed, atmospheric pressure, humidity and sun irradiation were simultaneously monitored using a dedicated station.

\section{METHODOLOGY}

\subsection{Pre-processing}

The selected frame rate of 2 seconds, for each independent band, created a temporal delay that produced a spatial displacement of moving objects between the bands. Using this configuration, leaves that moved with the wind were recorded in different pixel location in each band frame. Consequently, these

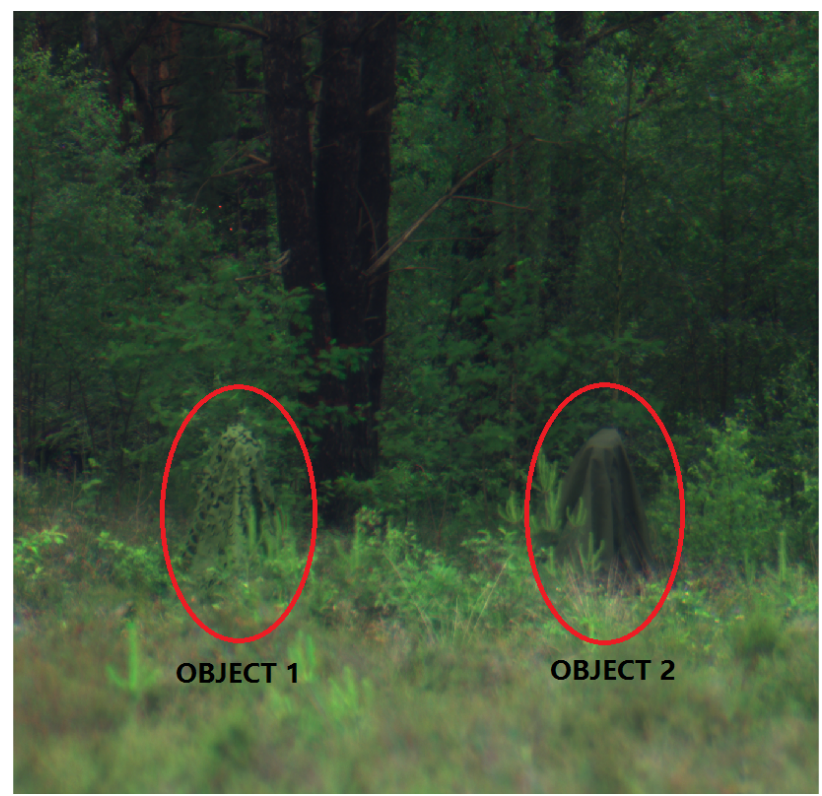

Figure 4. Concealed objects in a woodland scene

displacements created a situation where a single pixel contains reflectance that is correspondent to different materials along the wavelengths. To overcome this artifact, a spectral gradient analysis is used to mask out the pixels that are corresponded to moving objects. In general, a pixel with this characteristic has big differences in reflectance between adjacent bands, producing high spectral gradient values.

A transformed hyperspectral cube is then generated using the 1 st derivative of the spectrum $(\mathrm{Z})$ in each pixel, according to the following equation (1),

$$
Z^{\prime}=[z(2)-z(1) ; z(3)-z(2) ; \ldots ; z(m)-z(m-1)]
$$

where

$$
\begin{aligned}
& Z^{\prime}=\text { spectral gradient vector } \\
& z=\text { individual components of spectrum vector } Z \\
& m=\text { number of bands }
\end{aligned}
$$

In addition, a threshold on the spectral gradient vector is computed from a spectral reference library. The spectral vegetation library of the United States Geological Survey (USGS) (Kokaly et al., 2017) was chosen, from which vectors corresponding to trees, grass and leaves were selected and spectral gradients computed, as above. The resulting spectral gradient was then used as a threshold reference, to which the spectral gradient vector from each pixel in the acquired images were compared.

As shown in Figure 5 (top), when a derivative spectrum is within the threshold range, the pixel is retained. Otherwise, if a derivative spectrum is outside the threshold (Figure 5, bottom), the pixel is masked out and not further considered in the processing. The results of the gradient filter analysis for the vehicle scene are presented in Figure 6. It must be noticed that only pixels that contain moving background objects (i.e. leaves, in yellow) were masked out.

\subsection{Detection Algorithms}

Four supervised target detection methods were applied after implementing the above mentioned gradient filter. Specifically, the Constrained Energy Minimization (CEM), Adaptive 

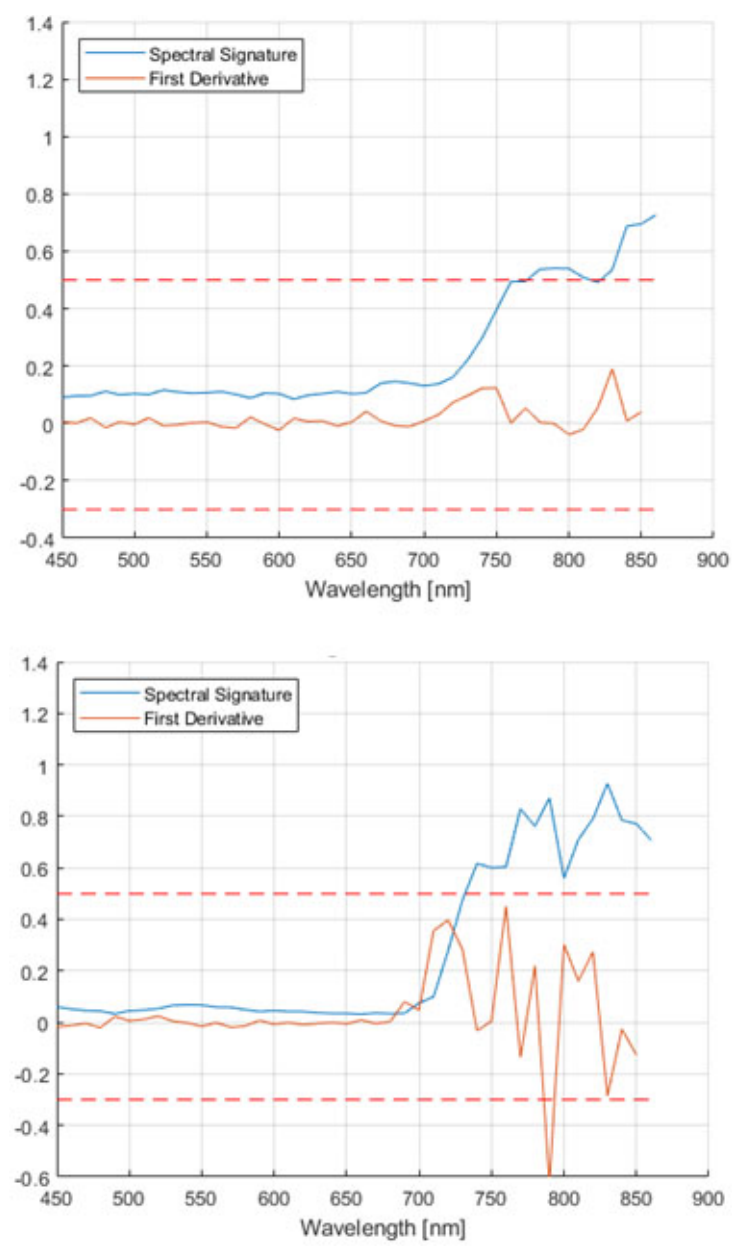

Figure 5. Mask using spectral gradient analysis. The original spectrum of a pixel (blue) and its derivative (orange)

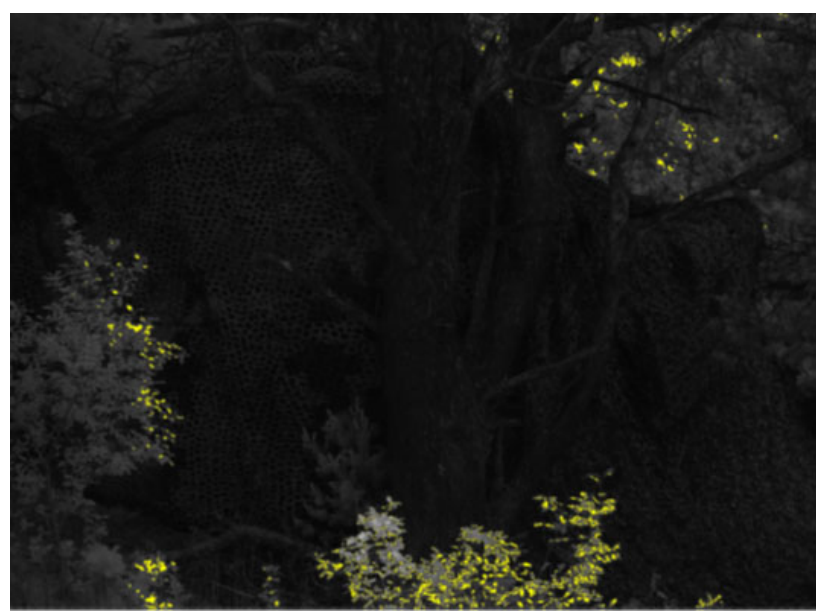

Figure 6. Results of the gradient filter; in yellow background pixels that were masked out.

Cosine Estimator (ACE), Adaptive Matched Subspace Detector (AMSD), and Orthogonal Subspace Projection (OSP) (Wang et al., 2016) were considered using reference spectra collected in Section 2.2 and random background pixels from the scene used as training or input.

\section{RESULTS}

\subsection{Concealed Vehicle}

The vehicle was concealed using two different camouflage nets (Figure 3). On the right side of the tree trunk, the vehicle is covered by a woodland net composed of $3 \mathrm{D}$ blending structures. On the left side, it is covered by printing fabric. The detection results of the 3D net are presented in Figure 7 and Figure 9 and the printing fabric in Figure 8 and Figure 10. ROC curves were generated using manualy labeled pixels.

The results show that in low-illumination conditions and with a low number of background pixels, the detection of the concealed targets is almost equally hard. The best detection score was achieved using the CEM for the printed fabric. However, in general, the performances of the CEM and the AMSD were quite similar in the detection of the two nets. These results are quite surprising as it can be expected that the detectors that require knowledge of background spectra from the scene (i.e. AMSD and OSP) will perform better than the ones that require only the target spectra. The results also show that the worse performances of ASP and ACE are mainly due to the confusion with the background materials, while the false alarms in the case of AMSD and OSP are due to confusion between the nets. In addition, results also show that it was easier to detect the printed fabric than the $3 \mathrm{D}$ net in this operational scene.
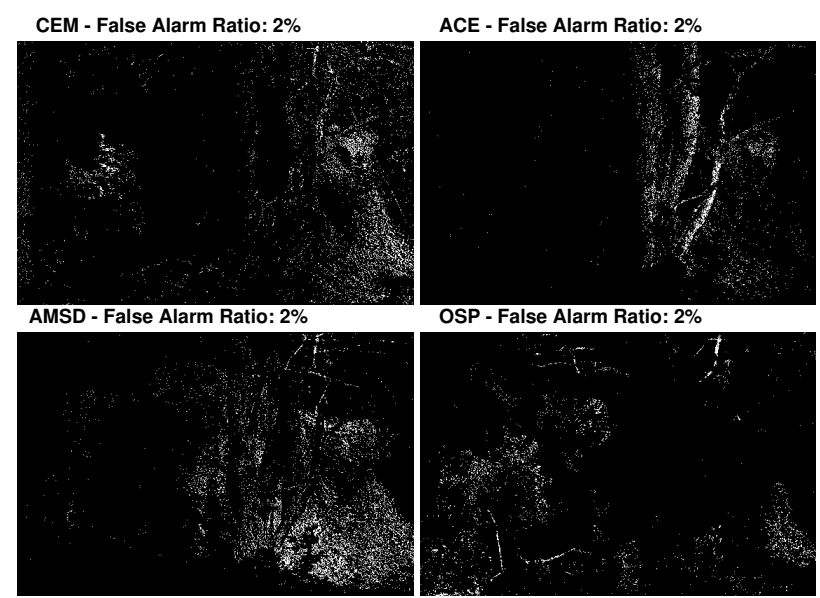

OSP - False Alarm Ratio: $2 \%$

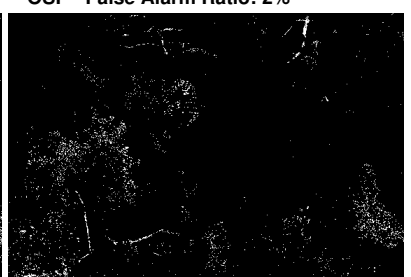

Figure 7. Detection results - concealed vehicle using 3D net
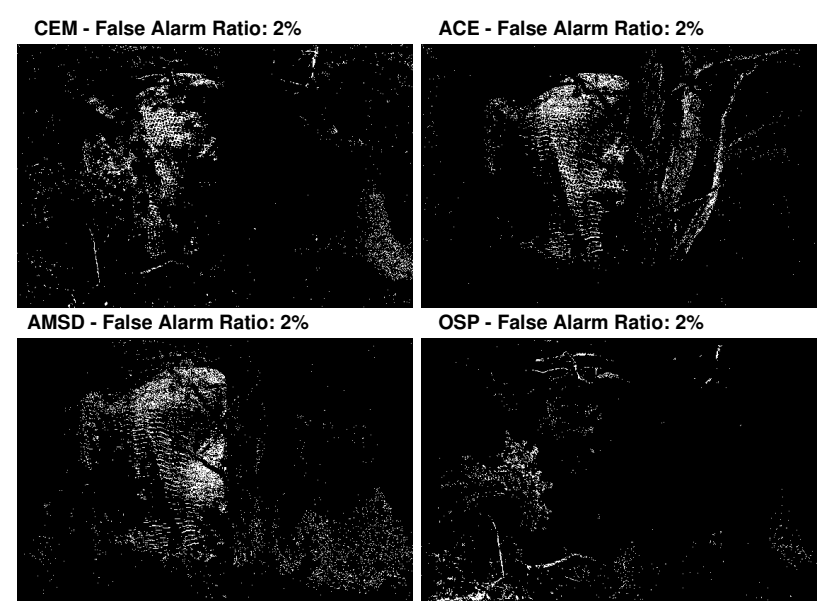

OSP - False Alarm Ratio: $2 \%$

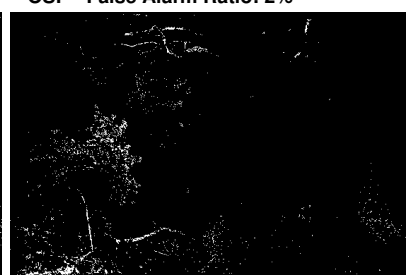

Figure 8. Detection results - concealed vehicle using printed net 


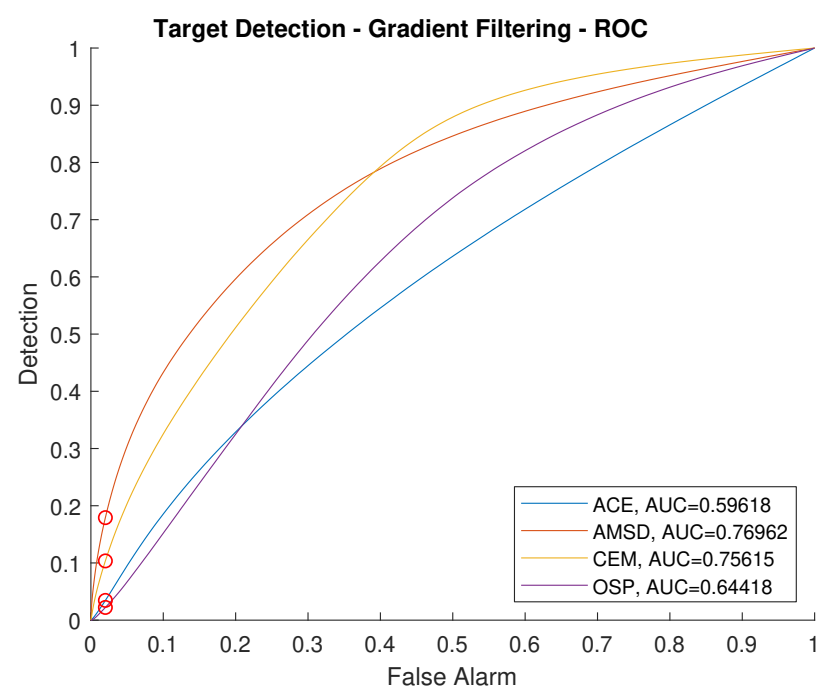

Figure 9. Detection of concealed vehicle - ROC of 3D net

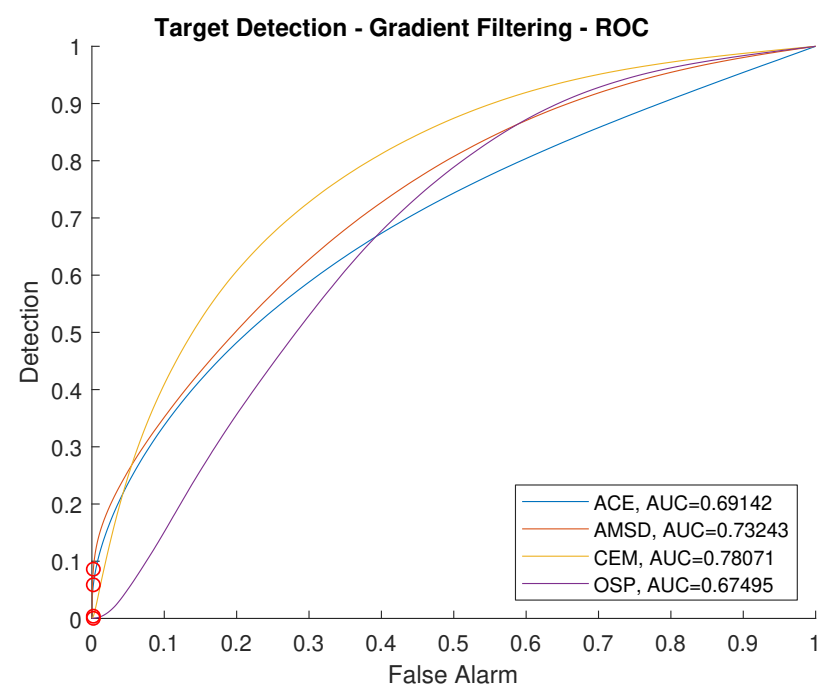

Figure 10. Detection of concealed vehicle - ROC of printed net

\subsection{Concealed Objects}

In the second scenario, equipment installed on tripods used as a heat source were covered with two different nets. The objects, labeled 1 and 2, were positioned in front of a North-European woodland (Figure 4) and scanned from a distance of about 200 meters. Object 1 was concealed with 3D net and Object 2 with printed net. The detection results and their correspondent ROCs are presented in Figures 11 and 12 for Object 1 and 13 and 14 for Object 2. It should be mentioned that these nets are different from the ones used to cover the vehicle.

The results show that it was relatively easy to detect concealed objects using Glana-L4 if the object is under direct illumination and not covered by trees. In general, as for the concealed vehicle, it was easier to detect an object concealed by a printed net than by a 3D net. Three out of the four detectors performed exceptionally well in detecting Object 2, except of the OSP. The confusion in the detection of the latter is mostly produced with Object 1 and the background. The detection performance of object-1 is less impressive and except of the CEM, the level of false alarms are quite high using any of the other three methods.
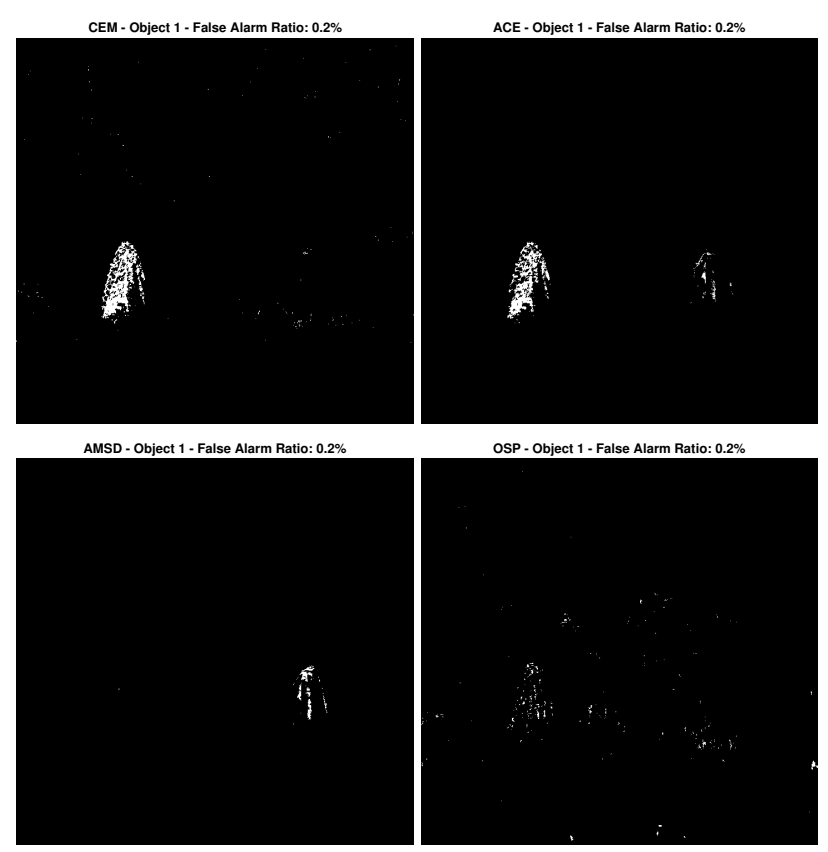

Figure 11. Object 1 target detection.

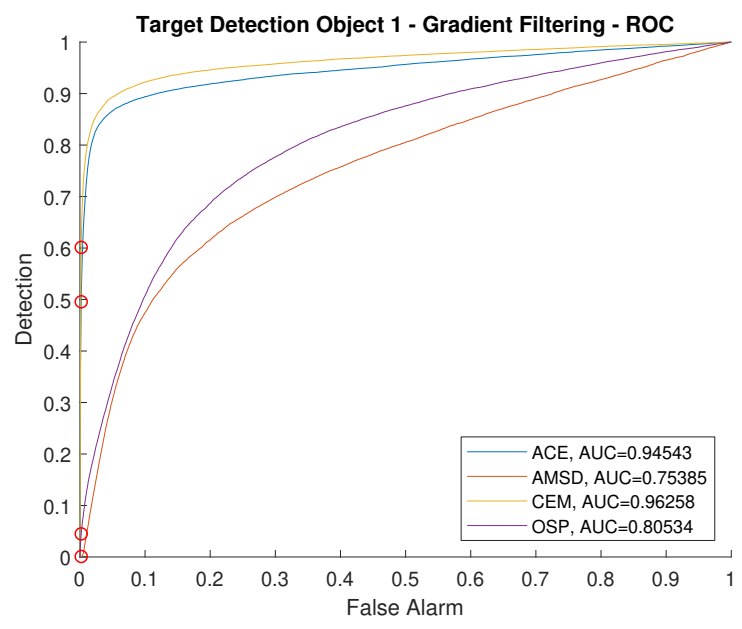

Figure 12. Object 1 ROC.

It is difficult to distinguish between object-1 and object-2.

\section{CONCLUSIONS}

In this paper, we presented an assessment of the capability of the imager L4 from Glana Sensors to detect concealed targets in woodland areas. The data set which comprises a collection of scenes acquired under various illumination conditions and containing different materials, revealed the potential this new sensing technology may bring to security operations. Specifically, the results show that it is relatively easy to detect small concealed objects under direct illumination and in front of the tree line, using the Glana-L4. It was harder to detect concealed vehicle under shadow and between the trees. Nevertheless, the detection results of the concealed vehicle were satisfactory using the CEM and the AMSD methods.

The obtained results also show that implementing a gradient filter to reduce displacement artifacts prior the detection significantly improved the results. Further experiments should be 

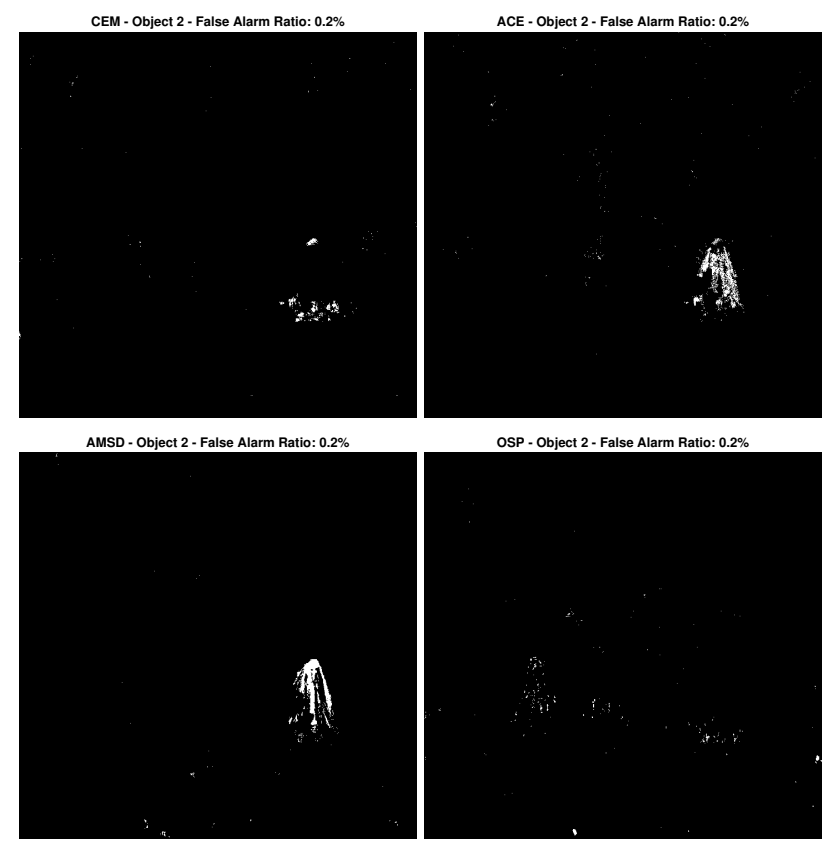

Figure 13. Object 2 target detection.

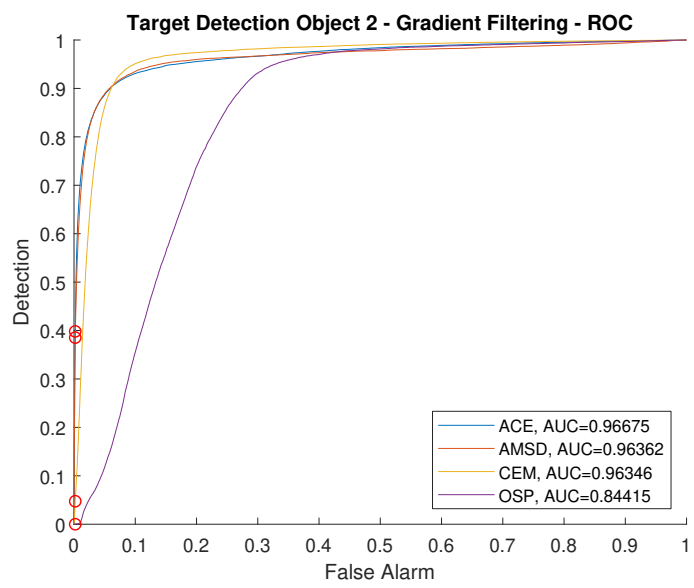

Figure 14. Object 2 ROC.

conducted in order to evaluate other target detection algorithms, particularly algorithms that do not require prior knowledge (i.e. anomaly detection) and algorithms that are exploiting both spatial and spectral information.

\section{ACKNOWLEDGEMENTS}

This research is conducted in the framework of EOXPOSURE project under grant agreement No 734541 of the Horizon 2020 research and innovation program (http://www.h2020eoxposure.eu/). It has been also supported by Junta de Extremadura (Decreto 14/2018, de 6 de febrero, por el que se establecen las bases reguladoras de las ayudas para la realizacion de actividades de investigacion y desarrollo tecnologico, and de divulgacion y de transferencia de conocimiento por los Grupos de Investigacion de Extremadura) under Grant GR18060.

\section{REFERENCES}

Ahlberg, J., Renhorn, I. G., Chevalier, T. R., Rydell, J., Bergström, D., 2017. Three-dimensional hyperspectral imaging technique. In Proc. SPIE 10198, Algorithms and Technologies for Multispectral, Hyperspectral, and Ultraspectral Imagery, XXIII.

Jafari, M., Sarkargar, A., Maghsoudi, Y., Valadan Zoej, M. J., 2014. Improving concealed target detection in forest area using fusion of Polarimetric SAR data and hyperspectral data using polarimetric and spectral signatures. Journal of Radar, 3(1), 13-23.

Kokaly, R., Clark, R., Swayze, G., Livo, K., Hoefen, T., Pearson, N., Wise, R., Benzel, W., Lowers, H., Driscoll, R., Klein, A., 2017. USGS Spectral Library Version 7. Data Series 1035.

Petra, A., Selina, G., Andreas, U., Gerald, K., Uli, R., 2018. Surveying forest stands and single trees using drones. FVA Annual Report 2018, 21-22.

Q. Ling, Y. Guo, Z. L. L. L. W. A., 2019. A Constrained SparseRepresentation-Based Binary Hypothesis Model for Target Detection in Hyperspectral Imagery. IEEE Selected Topics in Applied Earth Observations and Remote Sensing, 12(6), 19331947.

Renhorn, I. G., Axelsson, L., 2019. High spatial resolution hyperspectral camera based on exponentially variable filter. $O p$ tical Engineering, 58(10), 835-844.

Renhorn, I. G., Bergstrom, D., Hedborg, J., Letalick, D., Moller, S., 2016. High spatial resolution hyperspectral camera based on a linear variable Filter. Optical Engineering, 55(11).

Stefanou, M. S., Kerekes, J. P., 2010. Image-derived prediction of spectral image utility for target detection applications. IEEE Trans. Geosci. Remote Sens, 48(4), 1827-1833.

Waharte, S., Trigoni, N., 2018. Supporting Search and Rescue Operations with UAVs. 2010 International Conference on Emerging Security Technologies, 142-147.

Wang, G., Zhang, Y., He, B., Chong, K. T., 2016. A Framework of Target Detection in Hyperspectral Imagery Based on Blind Source Extraction. IEEE Journal of Selected Topics in Applied Earth Observations and Remote Sensing, 9(2), 835-844. 